\title{
Mechanical characteristics of the pulmonary artery in beagle dogs with hepatopulmonary syndrome and portopulmonary hypertension
}

\author{
GUOZHEN YAN, JUNFENG HE, YUELI YU, YANG LIU, YANFEN YUAN and ZHIYONG GUO
}

Department of Ultrasound, The First Affiliated Hospital of Baotou Medical College,

Baotou, Inner Mongolia Autonomous Region 014010, P.R. China

Received August 4, 2015; Accepted September 10, 2015

DOI: $10.3892 /$ br.2015.526

\begin{abstract}
The continuous changes in pulmonary hemodynamic properties in hepatopulmonary syndrome (HPS) and portopulmonary hypertension (PoPH) have not been fully characterized in large animal models of HPS and PoPH. Beagle dog models of HPS and PoPH were induced by chronic common bile duct ligation and Sephadex microspheres, respectively. The model was validated by catheter examination and pathological analyses, and the hemodynamic characteristics of the models were observed. The results revealed that the cross-sectional area of the blood vessel was significantly increased in HPS models, but it was significantly decreased in the PoPH models. Furthermore, the resistance of pulmonary circulation was elevated in models of HPS, but it was decreased in models of PoPH. The present findings renew the traditional view that pulmonary hypertension is due to the enhanced peripheral resistance.
\end{abstract}

\section{Introduction}

Liver transplantation (LT) has been performed since 1963 and is now a worldwide procedure (1). LT has a 30-day and 5-year mortality rate of 5 and $30 \%$, respectively (2). Abnormalities of the pulmonary vasculature found in association with coexisting chronic liver disease were first reported in the 1950s $(3,4)$. The pulmonary complications, such as hepatopulmonary syndrome (HPS) and portopulmonary hypertension (PoPH), make LT a procedure with more risks. Additionally, these liver disease complications reduce the chances of LT success, worsen the overall prognosis of patients and represent a challenge to the anesthesiologist $(5,6)$. There is limited information with

Correspondence to: Dr Guozhen Yan, Department of Ultrasound, The First Affiliated Hospital of Baotou Medical College, 41 Linyin Road, Baotou, Inner Mongolia Autonomous Region 014010, P.R. China

E-mail: guozhenyan2015@sina.com

Key words: beagle dog, hepatopulmonary syndrome, hemodynamic characteristics, portopulmonary hypertension regards to the epidemiology, pathophysiology and treatment of HPS and PoPH (1).

HPS and PoPH are associated with pulmonary arterial hypertension (PAH) and/or liver disease (7). HPS is the most common condition, identified in 5-30\% of cirrhosis patients, manifested by abnormal oxygenation due to excessive pulmonary microvascular dilation (8). HPS is a well-recognized cause of a poorer outcome in the liver disease patient. PoPH is characterized by the development of PAH in the setting of portal hypertension with or without hepatic disease (8). PoPH is observed in cirrhotic and non-cirrhotic portal hypertension and is not associated with the etiology of liver disease or the severity of portal hypertension. It is now clear that patients with PoPH or HPS should be properly diagnosed preoperatively and the severity of the disease should be graded to initiate the right treatment promptly (1). The proposed algorithm for diagnosing and grading HPS is based on the hemodynamic characteristics of pulmonary artery $(1,9)$. However, the mechanical characteristics of pulmonary artery with HPS and PoPH remain to be elucidated.

Therefore, the aim of the present study was to investigate the mechanical characteristics of HPS and PoPH in beagle dog.

\section{Materials and methods}

Animals. A total of 30 healthy beagle dogs (12 spayed females and 18 neutered males; median age, 3.2 years; range, 2.4-6.0; weight, $15 \mathrm{~kg}$ ), were used in the experiment. All the animals were kept in a clean level condition, with the room temperature maintained at $20 \pm 2^{\circ} \mathrm{C}$, relative humidity at $65 \pm 10 \%$, diurnal cycle and free access to standard food and tap water (10). Surgical procedures were performed under general anesthesia with medetomidine hydrochloride $(0.1 \mathrm{ml} / \mathrm{kg})$ and butorphanol tartrate $(0.1 \mathrm{ml} / \mathrm{kg})$, and under local anesthesia with lidocaine hydrochloride ( $2 \%$ with 1:80,000 epinephrine). Blood pressure, heart rate, respiratory rate and body temperature were closely monitored. Penicillin (400 U/g) was administered intramuscularly 3 consecutive days after the surgery to prevent infection.

The dogs were assigned to three groups randomly: HPS model, PoPH model and control. They had daily interaction with their caregivers and periodic access to outside 
playgrounds. Experimental protocols were approved by the Institutional Animal Care of Baotou Medical College (Inner Mongolia Autonomous Region, China).

Surgical and experimental procedures. Chronic common bile duct ligation (CBDL) in the animal is an established experimental model for human HPS (11). CBDL was performed for HPS model dogs as described previously (12). Normal control animals underwent mobilization of the common bile duct without ligation. Animals were evaluated 2 weeks after CBDL. All the animals had hepatic histological analysis and measurements of portal venous pressure and spleen weight (13). Lung tissues were obtained from each animal.

Sephadex microspheres (G50, 50-150 mm, medium; Pharmacia Biotech, Freiburg, Germany) were used to establish the experimental model for human PoPH, as previously described (10). In brief, $25 \mathrm{~g}$ microspheres were placed in $500 \mathrm{ml}$ of $0.9 \%$ saline (number of beads: $1.7 \times 10^{4} / \mathrm{ml}$ ), and subsequently the mixture was placed into an autoclave $\left(120^{\circ} \mathrm{C}\right.$ for $30 \mathrm{~min}$ ) for disinfection. Following disinfection, the size of the swollen beads should be 105-310 $\mu \mathrm{m}$. The emboli were kept in a $4^{\circ} \mathrm{C}$ refrigerator. Under an aseptic condition and through a midline abdominal incision, a catheter filled with heparin was inserted into the portal vein through the pancreaticoduodenal vein, with only its tip located in the main portal vein truck. The outer end was covered with a heparin lock and it was subsequently placed subcutaneously in the right hypogastrium (10). The dogs received intra-portal injection of Sephadex microspheres at a 5-day interval $(10 \mathrm{mg} / \mathrm{kg}$ body weight, mixed in $5 \mathrm{ml}$ saline, administered in $30 \mathrm{sec}$ ) 6 times in total; while the controls were administered the same volume of saline in an identical manner. In order to evenly distribute the microspheres in the liver, the body of the dog was gently turned whilst injecting the emboli (10). Following every injection, the catheter was washed with saline and subsequently filled with heparin to avoid occlusion (10).

Histological examination. The lung specimen of the main pulmonary artery was collected by thoracotomy subsequent to sacrificing the by intravenous injection of potassium chloride under anaesthetic. The specimens were fixed in formalin $(10 \%)$ and embedded in paraffin, sectioned (5-mm), stained with hematoxylin and eosin and examined by light microscopy $(10,14)$.

Hemodynamic characteristics of the main pulmonary artery. The pulmonary artery pressure (PAP) was measured using MedLab-U/4cs (Nanjing Medease Co., Ltd., Nanjing, China) every month after the initial CBDL or injection of the microspheres. The PAP prior to the CBDL or injection was regarded as the baseline. The level of the midaxillary line was set as the zero point (15) and subsequently the transducer was set to the same level to calibrate the machine prior to connecting to the catheter. The unit of measurement was $\mathrm{mmHg}$ $(1 \mathrm{mmHg}=0.133 \mathrm{kPa})$. The diameter and wall thickness of the pulmonary artery was measured by laparotomy. The velocity of blood flow in the pulmonary artery (PAV) was measured by the ALC-Ultrasonic blood flow system (Shanghai Alcott Biotech Co., Ltd., Shanghai, China). Pulmonary vascular resistance was calculated as PAP/PAV ( $\mathrm{mmHg} / \mathrm{l} / \mathrm{min})$. The ratio of the wall area and vascular cross-sectional area (Rwa-csa) of the main pulmonary artery was measured at the one-third position of the pulmonary valve side between the pulmonary valve and the bifurcation of pulmonary artery (16).

Statistical analysis. The measurements are expressed as mean \pm standard deviation. Statistical analysis was performed by SPSS 13.0 (SPSS, Inc., Chicago, IL, USA) for windows. Data were analyzed with the two-tailed Student's t-test or analysis of variance with Bonferroni correction for multiple comparisons between groups. $\mathrm{P}<0.05$ was considered to indicate a statistically significant difference.

\section{Results}

Pulmonary artery changes subsequent to CBDL and Sephadex microspheres injection. To assess whether the structure and hemodynamic characteristics of the pulmonary artery with HPS and PoPH changed, CBDL was used to establish the HPS model, and Sephadex microspheres injection was used to establish the PoPH model (Fig. 1). In the HPS model dog, the main pulmonary artery was enlarged and its wall became thin, the vascular diameter was larger, and the cross-sectional area was increased compared to the control (Fig. 1A and C). By contrast, in the PoPH model dog, the main pulmonary artery was smaller and its wall became thick, the vascular diameter was smaller, and the cross-sectional area was decreased compared to the control (Fig. 1B and C). Thus, HPS and PoPH had opposing changes in the morphology of the main pulmonary artery.

Hemodynamic characteristics of the main pulmonary artery. PAP of the control dogs was maintained on the baseline through the experiment (data not shown). In the HPS group, the systolic pressure and mean PAP were decreased (Fig. 2A). In the PoPH group, all the systolic pressure, diastolic pressure, and mean PAP increased (Fig. 2A). The pulmonary vascular resistance in the HPS group was also decreased, but was enhanced in the PoPH group (Fig. 2B).

Similar to the aforementioned observations, the diameter of the main pulmonary artery was enlarged in the HPS model dog, and was narrowed in the PoPH model dog (Fig. 3A). The wall thickness of the main pulmonary artery became thin in the HPS group, and thick in the PoPH group (Fig. 3B). Corresponding to those changes, the ratio of the wall area and vascular cross-sectional area (Rwa-csa) was significantly decreased in the HPS group, and significantly increased in the PoPH group (Fig. 3C).

\section{Discussion}

PoPH and HPS are frequent complications of portal hypertension and cirrhosis (1). In the present study, the PoPH and HPS models were established using beagle dogs with methods of CBDL and Sephadex microspheres injection. Arterial blood was drawn from the femoral artery as previously described (17). The alveolar-arterial oxygen gradient was calculated as $150-\left(\mathrm{PaCO}_{2} / 0.8\right)-\mathrm{PaO}_{2}(18)$. When the detection of $\mathrm{PaCO}_{2}$ was $<70 \mathrm{mmHg}$, the HPS model was effective. When there is portal hypertension, PAP is $>25 \mathrm{mmHg}$, 

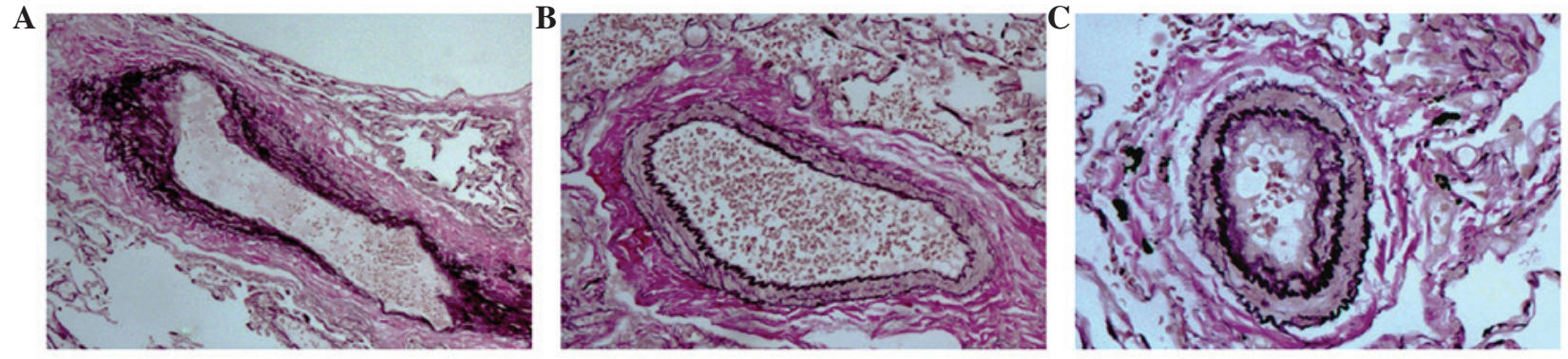

Figure 1. Histological examination of the main pulmonary artery. (A) Hepatopulmonary syndrome (HPS) model dog, (B) portopulmonary hypertension (PoPH) model dog, and (C) control dog. In the HPS model dog, the main pulmonary artery was enlarged and its wall became thin, the vascular diameter was larger, and the cross-sectional area was increased when compared to the control. By contrast, in the PoPH model dog, the main pulmonary artery was smaller and its wall became thick, the vascular diameter was smaller, and the cross-sectional area was decreased when compared to the control.
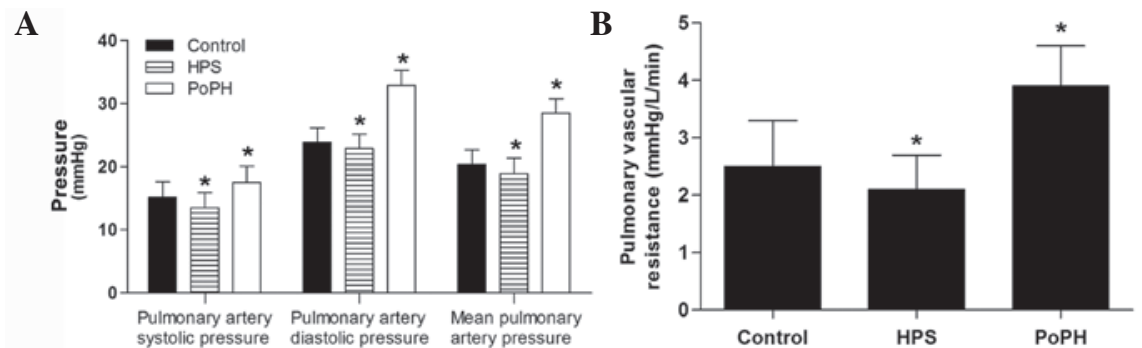

Figure 2. Changes in pressure and resistance of the pulmonary artery. (A) Systolic, diastolic and mean pulmonary artery pressures. (B) Pulmonary vascular resistance. ${ }^{*} \mathrm{P}<0.05$ vs. control. HPS, hepatopulmonary syndrome; PoPH, portopulmonary hypertension.
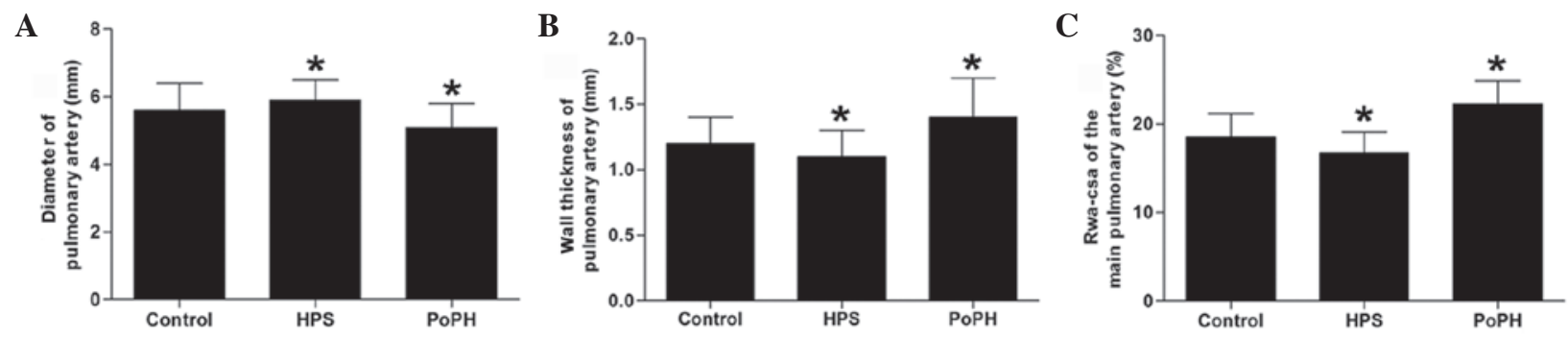

Figure 3. Change in the diameter of the pulmonary artery. The (A) diameter, (B) wall thickness and (C) ratio of the wall area and vascular cross-sectional area (Rwa-csa) of the main pulmonary artery. " $\mathrm{P}<0.05$ vs. control. HPS, hepatopulmonary syndrome; PoPH, portopulmonary hypertension.

pulmonary vascular resistance $>240$ dyn. $.8 e c . \mathrm{cm}^{-5}$ and pulmonary artery occlusion pressure $<15 \mathrm{mmHg}$, the $\mathrm{PoPH}$ model is successful. Subsequently, the hemodynamic characteristics of PoPH and HPS were investigated.

$\mathrm{PAH}$ is a set of diseases or clinical syndrome with a high incidence rate and mortality, which is characterized by an increase in PAP and pulmonary vascular resistance caused by etiology of vasoconstriction, vascular remodeling and thrombosis (19). The diagnostic criteria of PAH presented by the World Health Organization is mean PAP $>25 \mathrm{mmHg}$ in the resting state and $>25 \mathrm{mmHg}$ during exercise. When the PAH is not the correct treatment, it can cause right ventricular failure with increased volume load, and eventually can lead to fatality (20). Although the etiology of PAH has been investigated for $>100$ years and the quality of life has improved for certain patients, the pathogenesis of PAH remains elusive $(21,22)$. Current studies are focusing on the mechanism of PAH from a novel perspective to identify a promising target for treatment $(23,24)$. Currently, HPS and PoPH have become areas of interest worldwide $(1,18,25)$. HPS is a well-recognized cause of worsened outcome in the liver disease patient, which is defined by the combination of intrapulmonary vascular dilatation and hypoxemia in patients with chronic liver disease or portal hypertension (26). PoPH is the association between pulmonary hypertension and portal hypertension with or without hepatic disease (1). The hemodynamic monitor is particularly useful for the diagnosis of HPS and PoPH, and for the anesthesiologist in correct decision making (1).

In the present study, HPS and PoPH were further validated as different complications. In the HPS model dog, the main pulmonary artery was enlarged and its wall became thin, the vascular diameter was larger, and the cross-sectional area was increased when compared to the control (Fig. 1A and C). By contrast, in the PoPH model dog, the main pulmonary artery was smaller and its wall became thick, the vascular diameter was smaller, and the cross-sectional area was decreased when compared to the control (Fig. 1B and C). Therefore, the pulmonary morphology and hemodynamic properties of HPS and 
PoPH are changed in the opposing direction. As HPS and PoPH are observed in the same patients (27), we could hypothesize that there is a balance of hemodynamic properties and changes in the pulmonary artery morphology between HPS and PoPH, which may decide the development of lung complications. The present findings renew the traditional view that pulmonary hypertension is due to the enhanced peripheral resistance.

\section{Acknowledgements}

The present study was supported by the National Natural Science Foundation of China (grant no. 81160188).

\section{References}

1. Aldenkortt F, Aldenkortt M, Caviezel L, Waeber JL, Weber A and Schiffer E: Portopulmonary hypertension and hepatopulmonary syndrome. World J Gastroenterol 20: 8072-8081, 2014.

2. Dienstag JL and Cosimi AB: Liver transplantation - a vision realized. N Engl J Med 367: 1483-1485, 2012.

3. Mantz FA Jr and Craige E: Portal axis thrombosis with spontaneous portacaval shunt and resultant cor pulmonale. AMA Arch Pathol 52: 91-97, 1951.

4. Hoffbauer FW and Rydell R: Multiple pulmonary arteriovenous fistulas in juvenile cirrhosis. Am J Med 21: 450-460, 1956.

5. Fauconnet P, Klopfenstein CE and Schiffer E: Hepatopulmonary syndrome: The anaesthetic considerations. Eur J Anaesthesiol 30 721-730, 2013.

6. Krowka MJ: Management of pulmonary complications in pretransplant patients. Clin Liver Dis 15: 765-777, 2011.

7. Fritz JS, Fallon MB and Kawut SM: Pulmonary vascular complications of liver disease. Am J Respir Crit Care Med 187: 133-143, 2013.

8. Machicao VI, Balakrishnan M and Fallon MB: Pulmonary complications in chronic liver disease. Hepatology 59: 1627-1637, 2014.

9. Møller S, Henriksen JH and Bendtsen F: Extrahepatic complications to cirrhosis and portal hypertension: Haemodynamic and homeostatic aspects. World J Gastroenterol 20: 15499-15517, 2014.

10. Jin W, Deng L, Zhang Q, Lin D, Zhu J, Chen Y, Chen B and Li J: A canine portal hypertension model induced by intra-portal administration of Sephadex microsphere. J Gastroenterol Hepatol 25: 778-785, 2010.

11. Yang Y, Chen B, Chen Y, Zu B, Yi B and Lu K: A comparison of two common bile duct ligation methods to establish hepatopulmonary syndrome animal models. Lab Anim 49: 71-79, 2015.

12. Easter DW, Wade JB and Boyer JL: Structural integrity of hepatocyte tight junctions. J Cell Biol 96: 745-749, 1983.
13. Fallon MB, Abrams GA, Luo B, Hou Z, Dai J and Ku DD: The role of endothelial nitric oxide synthase in the pathogenesis of a rat model of hepatopulmonary syndrome. Gastroenterology 113: 606-614, 1997.

14. Yang W, Hu B, Wu W, Batra S, Blackburn MR, Alcorn JL, Fallon MB and Zhang J: Alveolar type II epithelial cell dysfunction in rat experimental hepatopulmonary syndrome (HPS). PLoS One 9: e113451, 2014.

15. Groszmann RJ, Glickman M, Blei AT, Storer E and Conn HO: Wedged and free hepatic venous pressure measured with a balloon catheter. Gastroenterology 76: 253-258, 1979.

16. Kasai H, Sugiura T, Tanabe N, Sakurai Y, Yahaba M, Matsuura Y, Shigeta A, Kawata N, Sakao S, Kasahara Y, et al: Electrocardiogram-gated 320-slice multidetector computed tomography for the measurement of pulmonary arterial distensibility in chronic thromboembolic pulmonary hypertension. PLoS One 9: e111563, 2014.

17. Fallon MB, Abrams GA, McGrath JW, Hou Z and Luo B: Common bile duct ligation in the rat: A model of intrapulmonary vasodilatation and hepatopulmonary syndrome. Am J Physiol 272: G779-G784, 1997.

18. Zhang J, Luo B, Tang L, Wang Y, Stockard CR, Kadish I, Van Groen T, Grizzle WE, Ponnazhagan S and Fallon MB: Pulmonary angiogenesis in a rat model of hepatopulmonary syndrome. Gastroenterology 136: 1070-1080, 2009.

19. Chan SY and Loscalzo J: Pathogenic mechanisms of pulmonary arterial hypertension. J Mol Cell Cardiol 44: 14-30, 2008.

20. Stenmark KR and McMurtry IF: Vascular remodeling versus vasoconstriction in chronic hypoxic pulmonary hypertension: A time for reappraisal? Circ Res 97: 95-98, 2005.

21. Zaiman A, Fijalkowska I, Hassoun PM and Tuder RM: One hundred years of research in the pathogenesis of pulmonary hypertension. Am J Respir Cell Mol Biol 33: 425-431, 2005.

22. Tabima DM and Chesler NC: The effects of vasoactivity and hypoxic pulmonary hypertension on extralobar pulmonary artery biomechanics. J Biomech 43: 1864-1869, 2010.

23. Dyer K, Lanning C, Das B, Lee PF, Ivy DD, Valdes-Cruz L and Shandas R: Noninvasive Doppler tissue measurement of pulmonary artery compliance in children with pulmonary hypertension. J Am Soc Echocardiogr 19: 403-412, 2006.

24. Rossmann JS: Elastomechanical properties of bovine veins. J Mech Behav Biomed Mater 3: 210-215, 2010.

25. Singh C and Sager JS: Pulmonary complications of cirrhosis. Medical Clin North Am 93: 871-883, viii, 2009.

26. Rodríquez-Roisin R, Krowka MJ, Hervé P and Fallon MB; ERS (European Respiratory Society) Task Force-PHD Scientific Committee: Highlights of the ERS Task Force on pulmonary-hepatic vascular disorders (PHD). J Hepatol 42: 924-927, 2005

27. Chung S, Lee K, Chang SA and Kim DK: Aggravation of hepatopulmonary syndrome after sildenafil treatment in a patient with coexisting portopulmonary hypertension. Korean Circ J 45: 77-80, 2015. 